\title{
A!
}

This is an electronic reprint of the original article.

This reprint may differ from the original in pagination and typographic detail.

Chen, Jingxue; Liu, Gao; Liu, Yining

\section{Lightweight Privacy-preserving Raw Data Publishing Scheme}

Published in:

IEEE Transactions on Emerging Topics in Computing

DOI:

10.1109/TETC.2020.2974183

Published: 01/12/2021

Document Version

Peer reviewed version

Please cite the original version:

Chen, J., Liu, G., \& Liu, Y. (2021). Lightweight Privacy-preserving Raw Data Publishing Scheme. IEEE

Transactions on Emerging Topics in Computing, 9(4), 2170-2174. https://doi.org/10.1109/TETC.2020.2974183

This material is protected by copyright and other intellectual property rights, and duplication or sale of all or part of any of the repository collections is not permitted, except that material may be duplicated by you for your research use or educational purposes in electronic or print form. You must obtain permission for any other use. Electronic or print copies may not be offered, whether for sale or otherwise to anyone who is not an authorised user. 


\title{
Lightweight Privacy-preserving Raw Data Publishing Scheme
}

\author{
Jingxue Chen, Gao Liu, and Yining Liu
}

\begin{abstract}
Data publishing or data sharing is an important part of analyzing network environments and improving the Quality of Service (QoS) in the Internet of Things (IoT). In order to incentives data providers (i.e., loT end-users) to contribute their data, privacy requirement is necessary when data is collected and published. In traditional privacy preservation techniques, such as k-anonymity, data aggregation and differential privacy, data is modified, aggregated, or added noise, the utility of the published data are reduced. Privacy-preserving raw data publishing is a more valuable solution, and $n$-source anonymity based raw data collection is most promising by delinking raw data and their sources. In this paper, a lightweight raw data collection scheme for publishing is proposed, in which the rawness and the unlinkability of published data are all really guaranteed with Shamir's secret sharing, and shuffling algorithm. Moreover, it is lightweight and practical for the loT environment by the performance evaluation.
\end{abstract}

Index Terms-data collection, privacy, rawness, unlinkability, lightweight

\section{INTRODUCTION}

W ITH the rapid development of the Internet of Thing (IoT), various IoT devices are used in many applications [1], such as smart grid [2], vehicle network [3], body area network [4]. These IoT devices really facilitate daily life, however, data privacy [5] [6] concerns should be addressed since the data from IoT devices consist of the sensitive information [7].

In past decades, k-anonymity [8] [9] and differential privacy [10] are widely researched to guarantee privacy when data is published. Specifically, k-anonymity guarantees that each person cannot be distinguished from at least other $k-1$ individuals by modifying corresponding attributes, meanwhile, differential privacy adds noise to the published data to avoid the disclosure of private information records.

However, both k-anonymity and differential privacy are used to protect the privacy of the data that has been collected and stored in data center, in fact, it is under the assumption that the data center is fully trusted since it owns or knows all stored data. However, the assumption that a data center or edge nodes connecting to IoT devices are fully trusted is not practical. Therefore, the edge nodes and data center should not directly obtain raw data from IoT devices, instead, the raw data collected with IoT devices should be masked before it is sent to other nodes. Two requirements are necessary, namely data privacy and utility.

Data aggregation allows a data center to obtain the average, maximum or minimum of data in an area without knowing individual data [11]. However, in some application scenarios, the average, maximum or minimum of data

This work was supported by the National Natural Science Foundation of China under grant no. 61662016, and Key projects of Guangxi Natural Science Foundation under grant no. 2018JJD170004.

Jingxue Chen and Yining Liu are with Guangxi Key Laboratory of Trusted Software, Guilin University of Electronic Technology, Guilin, 541004, China. Gao Liu is with State Key Laboratory on Integrated Services Networks and School of Cyber Engineering, Xidian University, Xi'an, 710126, China and with the Department of Communications and Networking, Aalto University, Espoo, Finland. (Corresponding author: Yining Liu, E-mail: ynliu@guet.edu.cn) cannot meet the needs, a variety of fine-grained data is required. Recently, a privacy-preserving computing function library is designed based on Intel Software Guard Extensions (SGX) [12]. However, Intel SGX may suffer from attack such as side-channel attack [13]. For data utilization, $n$ source anonymity is a feasible solution by delinking data and its source [14], where a piece of data is protected from an $n$-member group and simultaneously the rawness of data is ensured. Current $n$-source anonymity based raw data collection schemes mainly use virtual rings [15], a trusted third party (TTP) [14] and shuffling [16] to reserve slots for loading data. However, the sensitive data of an IoT device in virtual rings can be derived due to the collusion attack of its upstream and downstream devices. In addition, it is hard to deploy a TTP in practice. Hence, shuffle is used to replace the role of TTP, and simultaneously to ensure the rawness and unlinkability. Unfortunately, when $n$ IoT devices construct a group for masking their data, in [15] each IoT device of virtual ring reserves $n / 2$ slots on average, and in [14], [16], $n$ slots are reserved. As a consequence, the heavy storage cost is brought to each IoT device when $n$ is large.

In this paper, a lightweight raw data publishing scheme is proposed using secret sharing and shuffle, and two contributions are achieved as follows.

- Data privacy and utility are balanced by guaranteeing the unlinkability and the rawness.

- The lightweight requirement is achieved to make it more suitable for IoT devices.

The rest of this paper is organized as follows. Preliminaries are introduced in Section 2, problem definition is discussed in Section 3, our scheme and its analysis are presented in Section 4 and Section 5 respectively. Finally, the paper is concluded in Section 6. 


\section{Preliminaries}

\subsection{Bilinear Pairings}

The $p$ order cyclic additive group $G_{1}$, and $p$ order cyclic multiplicative group $G_{2}$, and a mapping $\hat{e}: G_{1} \times G_{1} \rightarrow$ $G_{2}$ ( $\hat{e}$ is a bilinear pairing) are defined. Assume that $P$ is a generator of $G_{1}$, which satisfies:

(1) Bilinearity: $\forall a, b \in Z_{p}^{*}, \hat{e}:(a P, b P)=\hat{e}(P, P)^{a b}$;

(2) Non-degeneracy: $\hat{e}:(P, P) \neq 1_{G_{1}}$;

(3) Computability: For any $P, Q \in G_{1}$, exists an efficient algorithm to compute $\hat{e}:(P, Q)$.

\subsection{Shuffle}

In [16], a shuffle algorithm is used to allocate slot locations, in which a ciphertext is re-randomized without changing the corresponding plaintext. The shuffle algorithm is defined as follows:

(1) Input $c_{i},(i \in[1, n])$ that is then encrypted into $c_{i}^{*}$, and rearranged using random permutation;

(2) Output the new permutated list.

More details can refer [16].

\subsection{Shamir's secret sharing}

In [17], assuming there are $n$ users $\left\{U_{1}, U_{2}, \ldots, U_{n}\right\}$, and a trusted dealer $D$.

Shares generation

$D$ chooses $t-1$ random numbers $a_{1}, a_{2}, \ldots, a_{t-1}$ and generates a polynomial $f(x)=s+a_{1} x+\cdots+a_{i} x^{i}+\cdots+$ $a_{t-1} x^{t-1}$ over a finite field $F_{p}$, where $p$ is a prime number, $s$ is a secret, and $t$ is a threshold value. $D$ sends $y_{i}=f\left(x_{i}\right)$ to $U_{i},(i \in[1, n])$ via a secure channel.

Secret reconstruction

The secret is recovered by computing

$$
s=f(0)=\sum_{i=1}^{t} \prod_{j=1, j \neq i}^{t} \frac{0-x_{j}}{x_{i}-x_{j}} y_{i} .
$$

\subsection{Secret sharing homomorphism}

Secret sharing homomorphism [18] is a useful tool for privacy-preserving computation. For example, in e-voting, an election center obtains the sum of voters' ballots without knowing individual ballots [19]. Assume there are two polynomials $f(x)$ and $g(x)$, and $s_{1}, s_{2}$ are their secrets to be shared respectively.

(1) Dealer $D$ sends $f(i)$ and $g(i)$ to the corresponding user $U_{i}$, where $i \in[1, n]$.

(2) $U_{i}$ computes and sends $f(i)+g(i),(i \in[1, n])$ to $D$.

(3) $D$ recovers the secret $s_{1}+s_{2}$ due to the additive homomorphism.

\section{PROBLEM DEFINITION}

\subsection{System model}

As shown in Fig.1, the system model consists of Cloud Server (CS), Fog Node (FN) and users $U_{i},(i \in[1, n])$ in group.

(1) CS sends a data collection request to $\mathrm{FN}$, then $\mathrm{FN}$ forwards the request to $U_{i},(i \in[1, n])$.

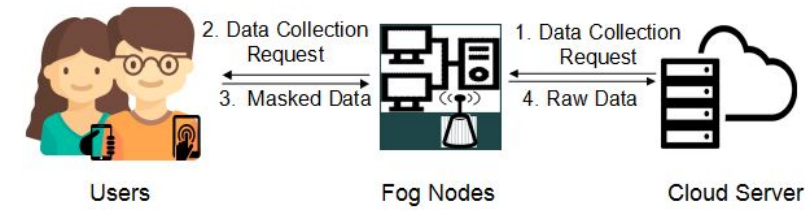

Fig. 1. System model

(2) After receiving the request, $U_{i},(i \in[1, n])$ collects and masks the data, then sends the masked data to FN.

(3) The raw data collected from $U_{i},(i \in[1, n])$ are extracted by $\mathrm{FN}$, then sent to $\mathrm{CS}$, meanwhile the relation between data and its source is privacy for all.

\subsection{Assumptions and threat model}

Only privacy issues are concerned under the assumption that secure communication channels have been established using cryptographic techniques among these entities [11] [16], and the semi-honest model is followed, i.e., CS, FN and users follow the protocols, meantime, they are curious to know the source of a piece of data.

- Data collectors (CS, FN) want to infer the data source from the information they received.

- Besides the data collectors, other users also attempt to collude to infer a user's data.

\section{OUR SCHEME}

Our scheme consists of Configuration Phase and Data Collection Phase. Details are as follows.

\subsection{Configuration phase}

\section{Key generation}

Step1: CS selects a security parameter $\gamma$ and generates $\left\{p, P, G_{1}, G_{2}, \hat{e}\right\}$, where $G_{1}, G_{2}$ are $p$ order cyclic groups, $P$ is a generator of $G_{1}$, and $\hat{e}$ is a bilinear pairing $\hat{e}: G_{1} \times G_{1} \rightarrow$ $G_{2}$.

CS chooses two secure cryptographic hash functions, $H$ : $Z_{p}^{*} \rightarrow Z_{p}^{*}$ and $H_{1}:\{0,1\}^{*} \rightarrow G_{1}$.

CS publishes $\left\{p, P, G_{1}, G_{2}, \hat{e}, H, H_{1}\right\}$.

Step2: Each entity selects a random number $s k_{\text {entity }} \in$ $Z_{p}^{*}$ as its secret key, then calculates and publishes $P K_{\text {entity }}=s k_{\text {entity }} P$.

Setup for data collection

$U_{i}$ selects $\beta_{i}$ partners in a group, generates a session key $k_{i j}$ with each partner $U_{j}$, and obtains its session list $\left\{k_{i 1}, k_{i 2}, \ldots, k_{i \beta_{i}}\right\},\left(\beta_{i} \in[1, n-1]\right),(i, j \in[1, n], i \neq j)$. For example, three users $\left\{U_{1}, U_{2}, U_{3}\right\}$ are in a group, $U_{1}$ and $U_{2}$ share the session key $k_{12}, U_{1}$ and $U_{3}$ share the session key $k_{13}$.

\subsection{Data collection phase}

Each user is assigned a position using shuffle [16], and this position corresponds to a coefficient in $n$-order degree polynomial $f(x)=a_{0}+a_{1} x+a_{2} x^{2}+\ldots+a_{n} x^{n}$, and the data from $U_{i}^{\prime} s$ is placed in this position. $U_{i}$ only knows its position, meanwhile knows nothing about others.

\section{Data collection initialization}


CS sends a data collection request Req = $\left\{I D_{C S}, I D_{F N}, T, \omega_{C S}\right\}$ to FNs, where $I D_{C S}$ and $I D_{F N}$ are identities of $\mathrm{CS}$ and $\mathrm{FN}, T$ is timestamp and $\omega_{C S}=s k_{C S} H_{1}\left(I D_{C S}\left\|I D_{F N}\right\| T\right)$.

\section{Forwarding request}

FN checks the timestamp $T$ and the equation $\hat{e}\left(\omega_{C S}, P\right)=\hat{e}\left(H_{1}\left(I D_{C S}\left\|I D_{F N}\right\| T\right), P K_{C S}\right)$. If yes, FN broadcasts the request.

\section{Masking raw data}

Step1: $U_{i},(i \in[1, n])$ generates its polynomial

$$
f_{i}(x)=a_{i, 0}+\lambda_{i, 1} x+\cdots+\left(R D_{i}+\lambda_{i, \ell}\right) x^{\ell}+\cdots+\lambda_{i, n} x^{n},
$$

where $a_{i, 0}$ is a random number selected by $U_{i}, R D_{i}$ represents the data from $U_{i}$, and the masking information $\lambda_{i, \ell}(\ell \in[1, n])$ is computed as follow:

$$
\lambda_{i, \ell}=\sum_{j=1, j \neq i}^{\beta_{i}}\left(I D_{i}-I D_{j}\right) H\left(k_{i j} \mid \ell\right) .
$$

Step2: $U_{i}$ generates $n+1$ shares $Y_{i, j}=f_{i}\left(I D_{j}\right),(j=$ $1, \ldots, n), Y_{i, F N}=f_{i}\left(I D_{F N}\right)$, where $I D_{i}$ is $U_{i}^{\prime} s$ identification. $U_{i}$ keeps $Y_{i, i}$ and sends $Y_{i, j}$ to $U_{j},(j=1, \ldots, n, j \neq i)$, sends $Y_{i, F N}$ and $a_{i, 0}$ to $F N$.

Step3: $U_{i}$ and FN obtain the masked data $M S D_{i}$ and $M S D_{F N}$ by adding the received shares.

Extracting raw data

FN checks if the equation

$\hat{e}\left(P, \sum_{i=1}^{n} \omega_{i}\right)=\prod_{i=1}^{n} \hat{e}\left(P K_{i}, H_{1}\left(M S D_{i}\left\|I D_{i}\right\| I D_{F N}\left\|T_{i}\right\| a_{i, 0}\right)\right)$

holds, then recoveries

$$
f(x)=a_{0}+a_{i} x^{i}+\cdots+a_{n} x^{n}
$$

using $\left(I D_{1}, M S D_{1}\right),\left(I D_{2}, M S D_{2}\right), \cdots,\left(I D_{F N}, M S D_{F N}\right)$.

$\left\{a_{1}, \ldots, a_{n}\right\}$ are collected by $\mathrm{FN}$, when the equation $a_{0}=\sum_{i=1}^{n} a_{i, 0}$ holds. Moreover, $\left\{a_{1}, \ldots, a_{n}\right\}$ are raw, which is not aggregated or averaged, not added noise, not modified. Nobody can link the element in two sets $\left\{a_{1}, \ldots, a_{n}\right\},\left\{U_{i}, \ldots, U_{n}\right\}$.

\section{An example of raw data collection}

Assume that $p=137, U_{1}, U_{2}, U_{3}$ and FN (their identifications are $1,2,3,4)$ collaborate to collect the heartbeats, $U_{1}, U_{2}, U_{3}$ correspond to three coefficients of a 3-degree polynomial after shuffling, such as $x^{2}, x$ and $x^{3}, U_{1}$ and $U_{2}$ share $k_{12}=2, U_{1}$ and $U_{3}$ share $k_{13}=3$.

$U_{1}$ calculates $Y_{1,1}=f_{1}(1), Y_{1,2}=f_{1}(2), Y_{1,3}=f_{1}(3)$ and $Y_{1, F N}=f_{1}(4)$, then keeps $Y_{1,1}$ secret and sends $Y_{1,2}$ to $U_{2}, Y_{1,3}$ to $U_{3}$, and $Y_{1, F N}$ to FN. The similar computation is for $U_{2}, U_{3}$. The shares received by each user and FN are listed in TABLE 1.

TABLE 1

The shares distribution

\begin{tabular}{|c|c|cccc|}
\hline \multicolumn{2}{|c|}{} & \multicolumn{4}{|c|}{ Receiver } \\
\cline { 3 - 6 } \multicolumn{2}{|c|}{} & $U_{1}$ & $U_{2}$ & $U_{3}$ & FN \\
\hline \multirow{3}{*}{ Sender } & $U_{1}$ & $Y_{1,1}$ & $Y_{1,2}$ & $Y_{1,3}$ & $Y_{1, F N}$ \\
& $U_{2}$ & $Y_{2,1}$ & $Y_{2,2}$ & $Y_{2,3}$ & $Y_{2, F N}$ \\
& $U_{3}$ & $Y_{3,1}$ & $Y_{3,2}$ & $Y_{3,3}$ & $Y_{3, F N}$ \\
\hline
\end{tabular}

$U_{i}$ computes its masked data $M S D_{i}=Y_{1, i}+Y_{2, i}+Y_{3, i}$, and sends $\left\{M S D_{i}, I D_{i}, I D_{F N}, T_{i}, a_{i, 0}\right\}$ to FN, where $T_{i}$ is a timestamp and $\omega_{i}=s k_{i} H_{i}\left(M S D_{i}\left\|I D_{i}\right\| I D_{F N}\left\|T_{i}\right\| a_{i, 0}\right)$, $(i=1,2,3)$.

Table 2 illustrates the following computation:

(1) The heartbeats of $U_{1}, U_{2}$ and $U_{3}$ are 78,60 and 85 respectively.

(2) $a_{1,0}=5, a_{2,0}=2$, and $a_{3,0}=1$.

(3) $\lambda_{1,1}=(1-2) \cdot H(2 \mid 1)+(1-3) \cdot H(3 \mid 1)=80$,

$\lambda_{1,2}=(1-2) \cdot H(2 \mid 2)+(1-3) \cdot H(3 \mid 2)=52$,

$\lambda_{1,3}=(1-2) \cdot H(2 \mid 3)+(1-3) \cdot H(3 \mid 3)=106$,

$\lambda_{2,1}=(2-1) \cdot H(2 \mid 1)=27$,

$\lambda_{2,2}=(2-1) \cdot H(2 \mid 2)=69$,

$\lambda_{2,3}=(2-1) \cdot H(2 \mid 3)=114$, and

$\lambda_{3,1}=(3-1) \cdot H(3 \mid 1)=30$,

$\lambda_{3,2}=(3-1) \cdot H(3 \mid 2)=16$,

$\lambda_{3,3}=(3-1) \cdot H(3 \mid 3)=54$.

(4) $94,24,34$ are the sum of the received shares of $U_{1}, U_{2}, U_{3}$, they are sent to $\mathrm{FN}$.

FN recovers the polynomial $f(x)=8+60 x+78 x^{2}+85 x^{3}$ $\bmod 137$, and ensures $\{60,78,85\}$ is the heartbeats set of $\left\{U_{1}, U_{2}, U_{3}\right\}$ when the equation $a_{1,0}+a_{2,0}+a_{3,0}=a_{0}$ holds, namely $5+2+1=8$.

\section{Analysis}

In this section, the proposed scheme is analyzed to really achieve the rawness and unlinkability. Moreover, the performance in terms of storage and computational burdens are evaluated, and the comparison with the excellent techniques are shown in TABLE 3.

\subsection{Security analysis}

\section{Rawness}

Raw data $R D_{i}$ can be obtained by reconstructing secrets on public shares.

Proof: Assuming that $U_{i}$ is assigned an unique coefficient of $x^{\ell},(l=1, \ldots, n)$ after shuffling, before no masking information is added, the polynomial is

$$
f_{i}(x)=a_{i, 0}+R D_{i} x^{\ell},(i=1, \ldots, n)
$$

Then, the set of coefficient of $a_{1}, \cdots, a_{n}$ of $\sum_{i=1}^{n} f_{i}(x)=$ $a_{0}+a_{1} x+a_{2} x^{2}+\cdots+a_{n} x^{n}$ corresponds the raw data $R D_{i}$, $(i=1, \ldots, n)$ from $U_{i},(i=1, \ldots, n)$.

When masking information is added, $U_{i}^{\prime} s$ polynomial is $f_{i}(x)=a_{i, 0}+\lambda_{i, 1} x+\cdots+\left(R D_{i}+\lambda_{i, \ell}\right) x^{\ell}+\cdots+\lambda_{i, n} x^{n}$, where $\lambda_{i, \ell}=\sum_{j=1, j \neq i}^{\beta_{i}}\left(I D_{i}-I D_{j}\right) H\left(k_{i j} \mid \ell\right)$ and $\sum_{i=1}^{n} \lambda_{i, \ell}=0$.

The shares of each user's polynomial are generated and sent to others, which guarantees that the sum of received shares $M S D_{1}, \ldots, M S D_{n}, M S D_{F N}$ of $U_{i},(i=1, \ldots, n)$ and $F N$ to correctly recover $a_{0}+a_{1} x+\cdots+a_{n} x^{n}$ that is same as Equation 5 using secret sharing homomorphism [18].

$\left\{a_{1}, \ldots, a_{n}\right\}$ are ensured to be the raw data from $\left\{U_{i},(i=1, \ldots, n)\right\}$ by FN when $a_{0}=\sum_{i=1}^{n} a_{i, 0}$.

\section{Unlinkability}

Nobody can link Raw data $R D_{i}$ and its source $U_{i},(i=$ $1, \ldots, n)$, even if one honest partner exists.

Proof: $U_{i}$ generates $n+1$ shares with its polynomial $f_{i}(x)=a_{i, 0}+\lambda_{i, 1} x+\cdots+\lambda_{i, \ell-1} x^{\ell-1}+\left(R D_{i}+\lambda_{i, \ell}\right) x^{\ell}+$ $\cdots+\lambda_{i, n} x^{n}$, where $n-1$ shares are sent to other users 
TABLE 2

An example over $F_{p}$, and $p=137$

\begin{tabular}{|l|cccccc|}
\hline & ID & polynomial & $p_{1}$ & $p_{2}$ & $p_{3}$ & FN \\
\hline$p_{1}$ & 1 & $f_{1}(x)=5+(80) x+(78+52) x^{2}+(106) x^{3}$ & $(1,47)$ & $(2,26)$ & $(3,30)$ & $(4,10)$ \\
$p_{2}$ & 2 & $f_{2}(x)=2+(60+27) x+69 x^{2}+114 x^{3}$ & $(1,135)$ & $(2,131)$ & $(3,126)$ & $(4,119)$ \\
$p_{3}$ & 3 & $f_{3}(x)=1+30 x+16 x^{2}+(85+54) x^{3}$ & $(1,49)$ & $(2,4)$ & $(3,15)$ & $(4,94)$ \\
\hline$M S D_{i}$ & & & $(1,94)$ & $(2,24)$ & $(3,34)$ & $(4,86)$ \\
\hline
\end{tabular}

in the group, and $a_{i, 0}$ and one share are sent to FN. In our hypothesis, there is at least one honest partner (except $U_{i}$ ) in the group who as a user will not betray $U_{i}$. Even if other $n-2$ users collude with FN to recover $f_{i}(x)$, however, $n$-degree polynomial cannot be recovered only with them, since at least $n+1$ points are necessary. Therefore, $f_{i}(x)$ is still unknown for all.

Furthermore, the masking information $\lambda_{i, \ell}=$ $\sum_{j=1, j \neq i}^{\beta_{i}}\left(I D_{i}-I D_{j}\right) H\left(k_{i j} \mid \ell\right)$ is still private, since at least one session key cannot be obtained by an adversary under the assumption that $U_{i}$ 's honest partner exist. Therefore, $U_{i}$ 's masking information, $\lambda_{i, 1}, \cdots \lambda_{i, \ell}, \cdots$, $\lambda_{i, n}$ is private even if $\beta_{i}-1$ partners collude with FN. As a consequence, the collected raw data $R D_{i}$ cannot be obtained by calculating $\left(R D_{i}+\lambda_{i, \ell}\right)-\lambda_{i, \ell}$, so that its privacy is preserved.

\subsection{Performance evaluation}

The performance of our scheme is evaluated and compared with [14] and [16].

\section{Storage burden}

Assume that the size of each data in [14] [16] is $L$ bits, the masked data $\Re_{i}=e_{i}^{1}\left|e_{i}^{2}\right| \ldots\left|e_{i}^{\text {slot }(i)} \oplus m_{i}\right| \cdots \mid e_{i}^{n}$ occupies $n L$ bits. When $n$ grows, the storage cost increases linearly. The masked data $M S D_{i}$ of user $U_{i}$ is the sum of all received shares, is only $L$ bits.

\section{Computational complexity}

Assume that there are $n$ users with an FN, and a simulation is executed using a laptop with Intel i5 $2.5 \mathrm{GHz}$ CPU and 8.00 GB memory, and computational complexity in shuffle and data collection is evaluated.

(1) Computational complexity in shuffle

The total computational complexity of shuffle is $\mathcal{O}(n \log p)$, since the shuffle technique adopts the ElGamal encryption [16]. $U_{i}$ encrypts its own pseudonym, shuffles a cipher list, and sends the new cipher list to its successor. The efficiency in shuffle depends on the length of pseudonym and users' position (i.e., the order in a transmission sequence) in a group. In our experiment, the position ranges from [200,1000], and the length of pseudonyms is 128 bits and 256 bits respectively. The result is shown in TABLE 4. When the group size is 1000 , the computation time of $U_{1000}$ is only $394 \mathrm{~ms}$.

(2) Computational complexity in data collection

Only the bilinear pairing operations are counted in data collection. Specifically, FN needs to execute two bilinear pairing operations to verify CS's data
TABLE 4

The computation time of shuffle (ms)

\begin{tabular}{|c|c|ccccc|}
\hline \multicolumn{2}{|c|}{} & \multicolumn{5}{c|}{ Group size } \\
\cline { 3 - 7 } \multicolumn{2}{|c|}{} & 200 & 400 & 600 & 800 & 1000 \\
\hline The length of & $128 b i t s$ & 106 & 176 & 248 & 320 & 388 \\
pseudonym & $256 b i t s$ & 120 & 187 & 262 & 321 & 394 \\
\hline
\end{tabular}

collection request, and $n+1$ bilinear pairing operation when checking the equation $\hat{e}\left(P, \sum_{i=1}^{n} \omega_{i}\right)=$ $\prod_{i=1}^{n} \hat{e}\left(P K_{i}, H_{1}\left(M S D_{i}\left\|I D_{i}\right\| I D_{F N}\left\|T_{i}\right\| a_{i, 0}\right)\right)$ to extract the raw data. Thus, FN takes $n+3$ [20] pairing operation. In addition, pairing operation execution $T_{\text {pair }}$ is about $2.187 \mathrm{~ms}$.

\subsection{Comparison}

Our scheme is compared with other well known privacy technique in TABLE 3, including TTP reliance, unlinkability, rawness, and computational complexity, etc.

\section{Conclusion}

In this paper, a lightweight raw data collection for data publishing is proposed based on secret sharing and shuffling algorithm, which is proved and evaluated to be more practical due to the efficiency and the privacy.

\section{REFERENCES}

[1] A. Zaslavsky, C. Perera, and D. Georgakopoulos, "Estimating age privacy leakage in online social networks," in proc. of ACC, 2012, pp. 21-29.

[2] Z. Erkin, J. R. Troncoso-Pastoriza, R. L. Lagendijk, and F. PérezGonzález, "Privacy-preserving data aggregation in smart metering systems: An overview," IEEE Signal Processing Magazine, vol. 30, no. 2, pp. 75-86, 2013.

[3] Y. Liu, S. Lv, M. Xie, Z. Chen, and P. Wang, "Dynamic anonymous identity authentication (daia) scheme for vanet," International Journal of Communication Systems, vol. 32, no. 5, p. e3892, 2019.

[4] M. Rabbi, S. Ali, T. Choudhury, and E. Berke, "Passive and insitu assessment of mental and physical well-being using mobile sensors," in proc. of UBIC, 2011, pp. 385-394.

[5] X. Li, Y. Zhu, J. Wang, Z. Liu, Y. Liu, and M. Zhang, "On the soundness and security of privacy-preserving svm for outsourcing data classification," IEEE Transactions on Dependable and Secure Computing, vol. 15, no. 5, pp. 906-912, 2018.

[6] Y. Zhu, Y. Zhang, X. Li, H. Yan, and J. Li, "Improved collusionresisting secure nearest neighbor query over encrypted data in cloud," Concurrency and Computation: Practice and Experience, $\mathrm{p}$. e4681, 2018. 
[7] R. Dey, C. Tang, K. Ross, and N. Saxena, "Estimating age privacy leakage in online social networks," in proc. of INFOCOM, 2012, pp. 2836-2840.

[8] P. Samarati and L. Sweeney, "Protecting privacy when disclosing information: k-anonymity and its enforcement through generalization and suppression," technical report, SRI International, Tech. Rep., 1998.

[9] L. Sweeney, "k-anonymity: A model for protecting privacy," International Journal of Uncertainty, Fuzziness and Knowledge-Based Systems, vol. 10, no. 05, pp. 557-570, 2002.

[10] C. Dwork, "Differential privacy," Encyclopedia of Cryptography and Security, pp. 338-340, 2011, doi:10.1007/978-1-4419-5906-5_752.

[11] Y. Liu, W. Guo, C.-I. Fan, L. Chang, and C. Cheng, "A practical privacy-preserving data aggregation (3pda) scheme for smart grid," IEEE Transactions on Industrial Informatics, vol. 15, no. 3, pp. 1767-1774, 2019.

[12] S. Li and K. Xue, "Secgrid: A secure and efficient sgx-enabled smart grid system with rich functionalities," CoRR, 2018. [Online]. Available: http://arxiv.org/abs/1810.01651

[13] Y. Wu, W. Zheng, B. Mao, and X. Wu, "Leaks or not: A framework for evaluating cache timing side channel attacks in sgx," in proc. of IEEE SmartWorld/SCALCOM/UIC/ATC/CBDCom/IO, 2018, pp. $1467-1470$.

[14] Y. Zhang, Q. Chen, and S. Zhong, "Privacy-preserving data aggregation in mobile phone sensing," IEEE Transactions on Information Forensics and Security, vol. 11, no. 5, pp. 980-992, 2016.

[15] M. Badra and S. Zeadally, "Design and performance analysis of a virtual ring architecture for smart grid privacy," IEEE Transactions on Information Forensics and Security, vol. 9, no. 2, pp. 321-329, 2014.

[16] Y. Liu, Y. Wang, X. Wang, Z. Xia, and J. Xu, "Privacy-preserving raw data collection without a trusted authority for iot," Computer Networks, vol. 148, pp. $340-348,2019$.

[17] A. Shamir, "How to share a secret," Communications of the ACM, vol. 22, no. 11, pp. 612-613, 1979.

[18] J. C. Benaloh, "Secret sharing homomorphisms: keeping shares of a secret secret (extended abstract)," in proc. of EUROCRYPT, 1987, pp. 251-260.

[19] Y. Liu and Q. Zhao, "E-voting scheme using secret sharing and k-anonymity," World Wide Web, vol. 22, no. 4, pp. 1657-1667, 2019.

[20] Y. Zhang, J. Zhao, D. Zheng, K. Deng, F. Ren, and X. Zheng, "Privacy-aware data collection and aggregation in iot enabled fog computing," in proc. of ICA3PP, 2018, pp. 581-590. 
TABLE 3

Comparison of mainstream privacy preservation techniques and our scheme

\begin{tabular}{|c|c|c|c|c|c|c|}
\hline & \multicolumn{6}{|c|}{ Mainstream privacy preservation techniques } \\
\hline & \multirow{2}{*}{$\frac{\text { k-anonymity }}{[9]}$} & \multirow{2}{*}{$\begin{array}{c}\text { Differential privacy } \\
{[10]}\end{array}$} & \multirow{2}{*}{$\frac{\text { Data aggregation }}{[11]}$} & \multicolumn{2}{|c|}{$n$-source anonymity } & \multirow[b]{2}{*}{ Our scheme } \\
\hline & & & & [14] & [16] & \\
\hline Data center & Fully trusted & Fully trusted & Semi-honest & Semi-honest & Semi-honest & Semi-honest \\
\hline TTP & NO & $\mathrm{NO}$ & NO & YES & NO & $\mathrm{NO}$ \\
\hline Rawness & NO & NO & $\mathrm{NO}$ & YES & YES & YES \\
\hline Unlinkability & YES & YES & YES & YES & YES & YES \\
\hline Masked data storage & - & - & 2 Lbits & $n$ Lbits & $n$ Lbits & Lbits \\
\hline $\begin{array}{l}\text { Computational complexity } \\
\text { of generating masked data }\end{array}$ & - & - & - & $\begin{array}{c}2 n \text { hashes } \\
2 n L \text {-bit XORs }\end{array}$ & $\begin{array}{c}n \beta_{i} \text { hashes } \\
n\left(\beta_{i}-1\right) L \text {-bit XORs }\end{array}$ & $\begin{array}{c}n \beta_{i} \text { hashes } \\
\text { secret shares generation }\end{array}$ \\
\hline $\begin{array}{c}\text { Computational complexity } \\
\text { of extracting raw data }\end{array}$ & - & - & - & $\begin{array}{l}(n-1) n L \\
\text {-bit XORs }\end{array}$ & $\begin{array}{l}(n-1) n L \\
\text {-bit XORs }\end{array}$ & Secret recovery \\
\hline
\end{tabular}

- this is not considered 